\title{
Constituent quark models for hadronic systems
}

\author{
Zahra Ghalenovi * \\ ${ }^{1}$ Department of Physics, Kosar University of Bojnord, Bojnord, Iran
}

\begin{abstract}
In this work we introduce two different potential models for hadronic systems such that the QCD concepts of the quark-quark and quarkantiquark interactions be satisfied. We present the simple methods to solve twoand three-body equation of meson and baryon systems respectively. The introduced models are studied in the relativistic and non-relativistic limits.
\end{abstract}

\section{Introduction}

One of the most important problems in modern physics is to determine the structure and properties of meson and baryon systems. Many potential models have been presented by authors to study the quarks dynamics and their properties [1-4]. The Dirac and Schrödinger equations are able to describe the behavior of quarks inside the hadrons in the relativistic and non-relativistic respectively. In this paper we present different methods to solve the Dirac and Schrödinger equations of the ground-state meson and baryon systems respectively for two different confining potentials. In both models, the spin-dependent potential is considered as a perturbation and the total energy of the hadron is obtained by a summation of the unperturbed $E_{0}$ and perturbed energy:

$$
E_{0}^{\text {total }}=E_{0}+\left\langle H_{s}\right\rangle
$$

where $H_{s}$ is the spin dependence interaction potential and is calculated using the unperturbed wave function as follows

$$
\left\langle H_{s}\right\rangle=\int d^{3} x \psi H_{s} \psi
$$

In Sect. 2, we present our method to solve two-body Dirac equation of meson systems and in Sect. 3, three-body Schrödinger equation is solved for baryon systems.

\section{Two-body Dirac equation as a potential model of mesons}

The Dirac equation for a quark of mass $m_{1}$ and energy $\varepsilon_{1}$ has the form

$$
\left.\left[\vec{\alpha} \cdot \overrightarrow{p_{1}}+\beta m_{1}+U_{01}(x)\right)\right] \psi_{1}(x)=\left(\varepsilon_{1}-V_{01}(x)\right) \psi_{1}(x),
$$

where $\psi_{1}(x)$ is the wave function of the quark and presented by

\footnotetext{
*e-mail: z_ghalenovi@kub.ac.ir
} 


$$
\psi_{1}(x)=\left(\begin{array}{l}
\chi_{1}(x) \\
\Theta_{1}(x)
\end{array}\right)
$$

By direct product of the wave function of the second particle (antiquark) $\psi_{2}(x)$ on both sides of Eq. (3) and substituting $\alpha$ and $\beta$ matrices into Eq. (3), results in (for details see Ref. [5])

$$
\Theta(x)=\frac{\left(\overrightarrow{\sigma_{1}} \cdot \overrightarrow{p_{1}}\right) \chi(x)}{\varepsilon_{1}+m_{1}},
$$

in which the direct product of the wave functions of two particles is chosen as the wave function of two-body meson system

$$
\psi(x)=\left(\begin{array}{l}
\chi(x) \\
\Theta(x)
\end{array}\right)
$$

We assume that the scalar potential is equal to the vector potential as

$$
2 U(x)=2 V(x)=a x-\frac{c}{x} .
$$

The potential (5) is the well-known Cornell potential. The first term $a x$ is a confining potential and the second term $-c / x$ is a Coulomb-like potential due to one-gluon exchange processes. $a$ and $c$ are positive constants. The variable $x$ is the relative quark-antiquark coordinate. Since the potential is assumed to depend on $x$ only, one can factor out the angular part of the two-body wave function $\psi(x)$.

For a two-body system, we assume that the particles have the identical mass $m$ and energy $\varepsilon$, where $m$ is the reduced mass of the particles (quark and antiquark)

$$
\left(p_{1}^{2}+p_{2}^{2}\right) \chi(x)=2\left(\varepsilon^{2}-m^{2}\right) \chi(x)-2\left(a x-\frac{c}{x}\right)(\varepsilon+m) \chi(x),
$$

where $\chi(x)$ is the top component of the wave function of the system. Using the operator form of the momentum we get

$$
-\left[\frac{d^{2}}{d x^{2}}+\frac{2}{x} \frac{d}{d x}-\frac{l(l+1)}{x^{2}}\right] \chi_{l}(x)=-\left(a x-\frac{c}{x}\right)\left(E_{l}+2 m\right) \chi_{l}(x)+\frac{1}{2}\left(E_{l}^{2}-4 m^{2}\right) \chi_{l}(x),
$$

where $l$ is the angular quantum number and $E_{l}=2 \varepsilon_{l}$ is the energy of the system.

The transformation

$$
\chi_{l}(x)=x^{-1} \varphi_{l}(x)
$$

reduces Eq. (7) to the form

$$
\frac{d^{2}}{d x^{2}} \varphi_{l}(x)+\left[\frac{1}{2}\left(E_{l}^{2}-4 m^{2}\right)-\left(E_{l}+2 m\right) a x+\frac{\left(E_{l}+2 m\right) c}{x}-\frac{l(l+1)}{x^{2}}\right] \varphi_{l}(x)=0 .
$$

The radial wave function $\varphi_{l}(x)$ is a solution of the reduced Schrödinger-type equation for the wave function of two identical particles with mass $m$ and interaction potential (5). We use a variational method to solve the equation for the case $l=0$ using the normalized test function [6]

$$
\varphi_{0}(x)=\sqrt{\frac{16 p^{3}}{\sqrt{2 \pi}}} x e^{-p^{2} x^{2}}
$$


where $p$ is the variational parameter. By minimization of the energy of the system, we calculate the energy and also $\chi(x)$ function.

Now by using the conservation of momentum $\vec{p}(x)=\vec{p}\left(r_{1}\right)+\vec{p}\left(r_{2}\right)$, where $\vec{p}(x)$ is the momentum of the meson system and regarding that the particles have the equal mass and equal energy, one can find the down component of the wave function as [5]

$$
\Theta_{0}(x)=\frac{\left(\overrightarrow{\sigma_{1}} \cdot \vec{p}_{1}\left(r_{1}\right)+\overrightarrow{\sigma_{2}} \cdot \vec{p}_{2}\left(r_{2}\right)\right)}{\left(E_{0}+2 m\right)} \chi_{0}(x)=\frac{(\vec{\sigma} \cdot \vec{p}(x))}{\left(E_{0}+2 m\right)} \chi_{0}(x)
$$

Using $(\vec{\sigma} \cdot \hat{x})(\vec{\sigma} \cdot \hat{x})=|\hat{x}|^{2}=1$ where $\hat{x}$ is the unit vector, we multiply the right-hand side of Eq. (11) with $(\vec{\sigma} . \hat{x})(\vec{\sigma} . \hat{x})$ and get

$$
\Theta_{0}(x)=\frac{(\vec{\sigma} \cdot \hat{x})}{\left(E_{0}+2 m\right)}[(\vec{\sigma} \cdot \hat{x})(\vec{\sigma} \cdot \vec{p}(x))] \chi_{0}(x)=\frac{(\vec{\sigma} \cdot \hat{x})}{\left(E_{0}+2 m\right)}\left[-i \frac{d}{d x}+i(\vec{\sigma} \cdot \vec{L})\right] \chi_{0}(x)
$$

For the ground state systems we have $l=0$, and therefore one can get the wave function of two-body meson system as follows

$$
\psi_{0}(x)=\left(\begin{array}{c}
\chi_{0}(x) \\
\frac{(\vec{\sigma} \cdot \hat{x})}{-i\left(E_{0}+2 m\right)} \frac{d \chi_{0}(x)}{d x}
\end{array}\right)=\left(\begin{array}{c}
x^{-1} \varphi_{0}(x) \\
\frac{(\vec{\sigma} \cdot \hat{x})}{-i\left(E_{0}+2 m\right)} \frac{d\left(x^{-1} \varphi_{0}(x)\right.}{d x}
\end{array}\right)
$$

\section{Three-body Schrödinger equation as a potential model of baryons}

In the quark model, a baryon is a three-body bound state made of quarks. The mathematical description of a three-body system is more complicated than that of a two-body system. In order to describe the baryon as a bound state of three constituent quarks, we define the configuration of three particles by two Jacobi coordinates $\rho$ and $\lambda$ as

$$
\vec{\rho}=\frac{1}{\sqrt{2}}\left(\vec{r}_{1}-\vec{r}_{2}\right), \quad \vec{\lambda}=\frac{1}{\sqrt{6}}\left(\vec{r}_{1}+\vec{r}_{2}-2 \vec{r}_{3}\right),
$$

such that

$$
m_{\rho}=\frac{2 m_{1} m_{2}}{m_{1} m_{2}}, \quad m_{\lambda}=\frac{3 m_{3}\left(m_{1}+m_{2}\right)}{2\left(m_{1}+m_{2}+m_{3}\right)} .
$$

Here $m_{1}, m_{2}$, and $m_{3}$ are the constituent quark masses. Instead of $\rho$ and $\lambda$, one can introduce hyperspherical coordinates [7-9] which are given by the angles $\Omega_{\rho}=\left(\theta_{\rho}, \phi_{\rho}\right)$ and $\Omega_{\lambda}=$ $\left(\theta_{\lambda}, \varphi_{\lambda}\right)$, respectively, together with the hyperradius $x$ and the hyperangle $\zeta$, defined by

$$
x=\sqrt{\vec{\rho}^{2}+\vec{\lambda}^{2}}, \quad \zeta=\arctan \left(\frac{\rho}{\lambda}\right) .
$$

Therefore, the Hamiltonian will be

$$
H=\frac{P_{\rho}^{2}}{2 m_{\rho}}+\frac{P_{\lambda}^{2}}{2 m_{\lambda}}+V(\rho, \lambda)=\frac{P^{2}}{2 m}+V(x) .
$$

In the hypercentral constituent quark model (HCQM), the quark potential $V$, is assumed to depend only on the hyperradius $x$. Therefore, in the three-quark wave function one can 
factor out the hyperangular part which is given by hyperspherical harmonics. The remaining hyperradial part of the wave function is determined by the hypercentral Schrödinger equation

$$
\left[\frac{d^{2}}{d x^{2}}+\frac{5}{x} \frac{d}{d x}-\frac{\gamma(\gamma+4)}{x^{2}}\right] \psi_{\gamma}(x)=-2 m\left[E_{\gamma}-V(x)\right] \psi_{\gamma}(x),
$$

where $\psi_{\gamma}(x), E_{\gamma}$, and $\gamma$ are the hyperradial part of the wave function, the energy eigenvalues, and the grand angular quantum number, respectively. The latter is given by $\gamma=2 \nu+l_{\rho}+l_{\lambda}$ where $l_{\rho}$ and $l_{\lambda}$ are the angular momenta associated with the $\rho$ and $\lambda$ variables and $v$ is a non-negative integer number. Here $m$ is the reduced mass,

$$
m=\frac{2 m_{\rho} m_{\lambda}}{m_{\rho}+m_{\lambda}} .
$$

We use the transformation

$$
\psi_{\gamma}(x)=x^{-5 / 2} \phi_{\gamma}(x)
$$

to bring equation 17 into the form

$$
\left[\phi_{\gamma}^{\prime \prime}(x)-\frac{(2 \gamma+3)(2 \gamma+5)}{4 x^{2}}\right] \phi_{\gamma}(x)=-2 m\left[E_{\gamma}-V(x)\right] \phi_{\gamma}(x) .
$$

For the baryon systems, we consider the harmonic oscillating potential as the confining interaction

$$
V(x)=a x^{2}-\frac{c}{x}
$$

Then we obtain the following equation

$$
\phi_{\gamma}^{\prime \prime}(x)+\left[-a_{1} x^{2}+\frac{c_{1}}{x}-\frac{(2 \gamma+3)(2 \gamma+5)}{4 x^{2}}\right] \phi_{\gamma}(x)=-\varepsilon_{\gamma} \phi_{\gamma}(x)
$$

where

$$
\varepsilon_{\gamma}=2 m E_{\gamma}, \quad a_{1}=2 m a, \quad c_{1}=2 m c .
$$

Here we take $a x^{2}$ as the parent and the Coulombic part is treated as perturbation (for details see Ref. [10]). Therefore, for the ground-state baryons the potential $V(x)$ turns exactly to the harmonic oscillator (H.O.) potential, and Eq. (22) reduces to

$$
\phi_{0}^{\prime \prime}(x)-\left[a_{1} x^{2}\right] \phi_{0}(x)=-\varepsilon_{0} \phi_{0}(x),
$$

The eigenfunction of the hypercentral H.O. potential are given by

$$
\psi_{0}(x)=N_{0} \exp \left(-\frac{m \omega x^{2}}{2}\right)
$$

and the eigenenergy is equal to $E_{0}=\frac{3}{2} \omega . N_{0}$ is the normalization constant and $\omega$ is the oscillation frequency $(\omega=\sqrt{2 a / m})$. In this model the perturbed energy of baryon system, is a summation of Coulombic and spin dependence interactions

$$
E_{0}^{\text {total }}=E_{0}+<-\frac{c}{x}>+<H_{s}>.
$$

One can use the method presented in Sect. 2 to solve the three-body Dirac problem of baryon system in a hypercentral approach. 


\section{References}

[1] D. Ebert, R. N. Faustov, V. O. Galkin and A. P. Martynenko, Phys. Rev. D 66, 014008 (2002)

[2] J. -R. Zhang and M. -Q. Huang, Phys. Rev. D 78, 094015 (2008)

[3] C. Albertus, J. Nieves, J. M. Verde-Velasco, E. Hernandez, Eur. Phys. J. A 32, 183 (2007)

[4] A. Valcarce, H. Garcilazo and J. Vijande, Phys. Rev. C 72, 025206 (2005)

[5] H. Hassanabadi, A. A. Rajabi, Few-Body Syst. 41, 201 (2007)

[6] Z. Ghalenovi, F. Giacosa and D. H. Rischke, Acta Phys. Polon. B 47, 5 (2016)

[7] Z. Ghalenovi and M. Moazzen Sorkhi, Eur. Phys. J. Plus 133, 301 (2018)

[8] Z. Ghalenovi and M. Moazzen, Eur. Phys. J. Plus 132, 8 (2017)

[9] Z. Ghalenovi, A. A. Rajabi, and A. Tavakolinezhad, Int. J. Mod. Phys. E 21, 6 (2012)

[10] I. J. R. Aitchison and J. J. Dudek, Eur. J. Phys. 23, 605 (2002) 